\title{
20. OXYGEN AND CARBON ISOTOPE STRATIGRAPHY FOR THE QUATERNARY OF HOLE 502B: EVIDENCE FOR TWO MODES OF ISOTOPIC VARIABILITY ${ }^{1}$
}

\author{
Warren L. Prell, Department of Geological Sciences, Brown University, Providence, Rhode Island
}

\begin{abstract}
The oxygen and carbon isotopic record of $G$. sacculifer in DSDP Holes 502 and 502B shows that the Quaternary interval is almost complete. A slight hiatus exists in the upper Matuyama chron. The $\delta^{18} \mathrm{O}$ data show two modes of isotopic variation: depleted values and low variability in the early Quaternary (910-1660 k.y.) and enriched composition and high variability in the late Quaternary ( $0-730 \mathrm{k} . \mathrm{y}$.). These data document the change in mean composition between the early and late Quaternary, which occurs about $900 \mathrm{k} . y$. ago. These two modes of $\delta^{18} \mathrm{O}$ variation reflect the changing ice budget on the continents and imply different envelopes of sea level variation during the early and late Quaternary. The occurrence of these two modes implies that boundary conditions during the early Quaternary were significantly different from those of the late Quaternary.
\end{abstract}

\section{INTRODUCTION}

One of the studies made possible by hydrologic piston coring at Site $\mathbf{5 0 2}$ is a detailed examination of the oxygen and carbon isotopic stratigraphy in the Quaternary of the Caribbean Sea. Prior to Leg 68, no conventional piston corer had penetrated the Brunhes boundary in Caribbean sediments. A few cores had recovered the entire Quaternary, but drilling disturbance (e.g., Hole 154A) prevented detailed analyses. In fact, in the entire world ocean only two piston cores (V16-205, Atlantic; V28-239, western Pacific) contain a relatively complete biotic, paleomagnetic, and isotopic stratigraphy of the Quaternary. However, slow rates of accumulation $(\leq 1 \mathrm{~cm} / \mathrm{k} . \mathrm{y}$.) and bioturbation in these cores combine to produce relatively low-amplitude $\delta^{18} \mathrm{O}$ fluctuations, especially in the early Quaternary. Shackleton and Opdyke (1976) noted that "Because of the effects of mixing, it is not possible to say whether the true amplitude of glacial-interglacial change was smaller in midMatuyama time."

The objective of this study is to provide a detailed isotopic record for the entire Quaternary in sediments which have accumulated rapidly enough to minimize the problem of mixing. The record at Site 502 represents the first detailed stratigraphy of the entire Quaternary in Caribbean sediments. Hole 502B was selected for the Quaternary isotopic record because it has the most complete recovery and least disturbance. Sections of Hole 502 were used to fill gaps in the recovery of Hole 502B. In this chapter I present the basic stratigraphy for the Quaternary section and compare it to other published stratigraphies.

\section{PALEOMAGNETIC STRATIGRAPHY}

The detailed magnetic polarity stratigraphy of Hole 502B is summarized in Kent and Spariosu (this volume).

${ }^{1}$ Prell, W. L., Gardner, J. v., et al., Init. Repts. DSDP, 68: Washington (U.S. Govt. Printing Office).
The location and "corrected sub-bottom depth" of the major paleomagnetic boundaries in the Quaternary section are as follows: Brunhes/Matuyama, Samples 502B$5-1,140-150 \mathrm{~cm}(16.20-16.30 \mathrm{~m})$; top of the Jaramillo, 502B-5-3, $110 \mathrm{~cm}$ (18.62 m); bottom of the Jaramillo, 502B-6-2, $45-70 \mathrm{~cm}(21.31-21.56 \mathrm{~m})$; top of the Olduvai was not recovered; bottom of the Olduvai, 502B-11-1, $90-110 \mathrm{~cm}(42.23-42.43 \mathrm{~m})$. On the basis of overlapping isotopic records from Hole 502B (Cores 8 and 9) and Hole 502 (Cores 9 and 10) which contained the top of the Olduvai, I placed the top of the Olduvai at $\mathbf{3 7 . 5}$ meters in Hole 502B. A summary of the paleomagnetic stratigraphy from the Olduvai subchron through the Brunhes chron is shown in Figure 1.

\section{ISOTOPIC RECORD OF THE QUATERNARY}

Oxygen-isotope stratigraphy of deep sea sediments has become the standard against which other stratigraphies are compared, especially in the late Pleistocene (Brunhes Chron). A number of studies (Shackleton, 1967; Duplessy et al., 1970; Shackleton and Opdyke, 1973) have demonstrated that the $\delta^{18} \mathrm{O}$ variation in pelagic deep sea sediments is predominantly a measure of global ice volume. The $\delta^{18} \mathrm{O}$ variations are global in extent and synchronous within the mixing time of the ocean and minor modification by bioturbation (Shackleton and Opdyke, 1973). The fast accumulation rate and low disturbance of Hole 502B make it an ideal section for establishing a detailed isotopic stratigraphy of the Quaternary sediments of the Atlantic-Caribbean.

\section{ANALYTICAL METHODS}

We measured the $\delta^{18} \mathrm{O}$ and $\delta^{13} \mathrm{C}$ of Globigerinoides sacculifer $(300-355 \mu \mathrm{m})$ at $10-\mathrm{cm}$ intervals from the core top through Core 6 $(23.65 \mathrm{~m})$ and at $20-\mathrm{cm}$ intervals from Cores 502B-6 through 502B-11 $(35.84 \mathrm{~m})$. Cores $502-9$ and $502-10$ were analyzed at 20 -cm intervals. Approximately 20 individuals of each species were picked from each sample and cleaned in ultrasonic bath to remove fine-fraction contamination. All samples were roasted under vacuum at $370^{\circ} \mathrm{C}$ for one hour. $\mathrm{H}_{2} \mathrm{O}$ and $\mathrm{CO}_{2}$ were extracted from the carbonate reaction with orthophosphoric acid at $50^{\circ} \mathrm{C}$, separated by a series of three freezing 


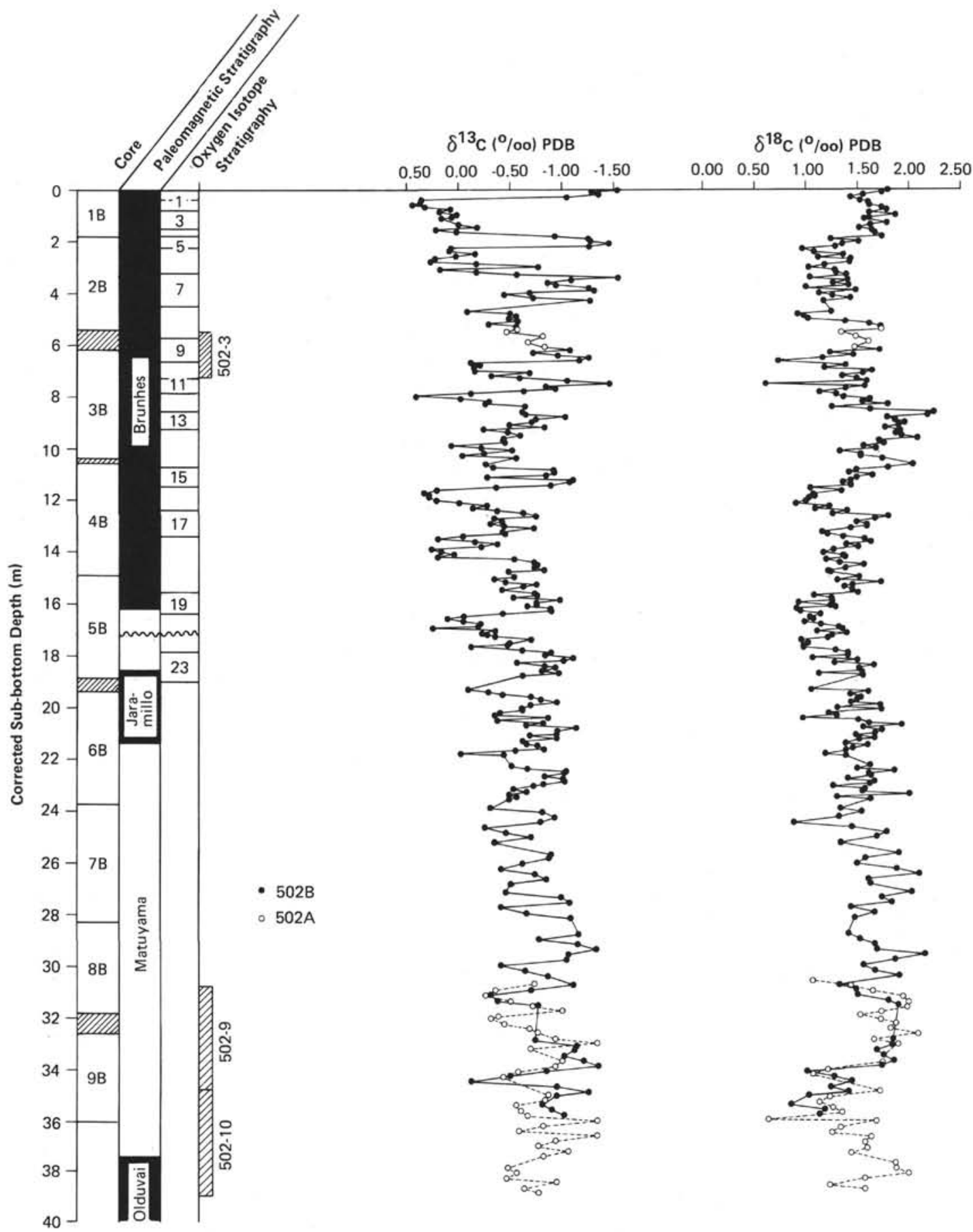

Figure 1. Summary of the magneto- and isotopic stratigraphy of Hole 502B. Sub-bottom depths are corrected for voids and allochthonous drilling mud at the top of cores. The isotopic data in Hole 502 have been adjusted to fit the depth scale of Hole 502B. Isotopic measurements are on Globigerinoides sacculifer (300-355 $\mu \mathrm{m})$. Where duplicates are present, average values are plotted. All data are given in Table 1.

transfer steps, and the $\mathrm{CO}_{2}$ was analyzed in an on-line VG Micromass $602 \mathrm{D}$ mass spectrometer. All data are referred to PDB by the standard $\delta$ notation (Craig, 1957). Calibration to PDB is through three intermediate laboratory standards. Agreement among all calibration standards is $\pm 0.2 \%$. The analytical precision, indicated by the first acceptable analysis of the working carbonate standard before each analytical session (41 days), is $\pm 0.092 \%$ ( $1 \sigma$ ) for oxygen and $\pm 0.086 \%$ $(1 \sigma)$ for carbon. Analytical precision based on 32 blind duplicate analyses run on separate days is $\pm 0.10 \%$ (average $1 / 2 \Delta \delta^{18} \mathrm{O}$ ) and $\pm 0.05 \%$ (average $1 / 2 \Delta \delta^{13} \mathrm{C}$ ). The data (averages of duplicates) are plotted in Figure 1, and all data are summarized in Table 1. After cor- relation to Hole 502B, data for Hole 502 are plotted on the 502B depth scale.

\section{Late Quaternary Isotopic Records}

The concept of oxygen isotope stages was established by Emiliani (1955) and later expanded by Shackleton and Opdyke $(1973,1976)$ and van Donk (1976). The stages are numbered in the direction of increasing age, with odd-numbered stages representing depleted values 
Table 1. Oxygen and carbon isotopic data for Holes 502 (Cores 3, 9, and 10) and 502B (Cores 1 through 9). All measurements are on $G$. sacculifer (300-355 $\mu \mathrm{m})$. On the basis of blind duplicates, analytical precision is $\pm 0.10 \%$ 。 (average $1 / 2 \Delta$ ) for $\delta^{18} \mathrm{O}$ and $\pm 0.05 \%$ for $\delta^{13} \mathrm{C}$.

\begin{tabular}{|c|c|c|c|c|}
\hline \multirow[b]{2}{*}{$\begin{array}{c}\text { Sample } \\
\text { (level in } \mathrm{cm} \text { ) }\end{array}$} & \multirow{2}{*}{$\begin{array}{l}\text { Corrected } \\
\text { Sub-bottom } \\
\text { Depth (m) }\end{array}$} & \multirow{2}{*}{$\begin{array}{c}\text { Coarse } \\
\text { Fraction } \\
(\%)\end{array}$} & \multicolumn{2}{|c|}{ G. sacculifer } \\
\hline & & & $\begin{array}{l}{ }^{18} \mathrm{O} \\
(\%)\end{array}$ & $\begin{array}{l}{ }^{13} \mathrm{C} \\
(\%)\end{array}$ \\
\hline \multicolumn{5}{|l|}{ Hole 502B } \\
\hline $1-1,2.5$ & 0.025 & 32.4 & -1.54 & 1.80 \\
\hline $1-1,10.5$ & 0.105 & 37.5 & -1.29 & 1.74 \\
\hline $1-1,20.0$ & 0.200 & 44.1 & -1.36 & 1.56 \\
\hline $1-1,30.5$ & 0.305 & 34.9 & -1.05 & 1.44 \\
\hline $1-1,43.0$ & 0.430 & 19.9 & 0.35 & 1.53 \\
\hline $1-1,51.5$ & 0.515 & 14.5 & 0.36 & 1.60 \\
\hline $1-1,61.0$ & 0.590 & 15.4 & 0.44 & 1.62 \\
\hline $1-1,71.5$ & 0.695 & 15.4 & 0.32 & 1.74 \\
\hline $1-1,81.0$ & 0.790 & 16.6 & 0.07 & 1.79 \\
\hline $1-1,91.5$ & 0.895 & 18.2 & 0.13 & 1.50 \\
\hline $1-1,91.5$ & 0.895 & 18.2 & 0.24 & 1.75 \\
\hline $1-1,101.0$ & 0.990 & 18.0 & 0.01 & 1.87 \\
\hline $1-1,111.5$ & 1.095 & 27.2 & 0.06 & 1.62 \\
\hline $1-1,114.0$ & 1.120 & 26.3 & 0.16 & 1.57 \\
\hline $1-1,131.5$ & 1.295 & 32.0 & 0.0 & 1.79 \\
\hline $1-1,139.0$ & 1.370 & 17.4 & -0.01 & 1.63 \\
\hline $1-2,2.5$ & 1.485 & 31.5 & -0.39 & 1.52 \\
\hline $1-2,2.5$ & 1.485 & 31.5 & 0.01 & 1.53 \\
\hline $1-2,11.0$ & 1.570 & 18.3 & 0.21 & 1.64 \\
\hline $1-2,20.5$ & 1.665 & 22.1 & 0.01 & 1.67 \\
\hline $2-1,12.5$ & 1.815 & 30.4 & -0.94 & 1.74 \\
\hline $2-1,22.0$ & 1.910 & 18.3 & -1.26 & 1.25 \\
\hline $2-1,32.5$ & 2.015 & 26.3 & -1.28 & 1.52 \\
\hline $2-1,41.0$ & 2.100 & 33.8 & -1.46 & 1.36 \\
\hline $2-1,51.5$ & 2.205 & 34.4 & -1.27 & 1.29 \\
\hline $2-1,61.0$ & 2.300 & 25.7 & 0.06 & 0.97 \\
\hline $2-1,71.5$ & 2.405 & 16.1 & 0.08 & 1.08 \\
\hline $2-1,83.0$ & 2.520 & 15.5 & -0.17 & 1.37 \\
\hline $2-1,91.5$ & 2.595 & 8.9 & 0.02 & 1.13 \\
\hline $2-1,101.0$ & 2.700 & 9.1 & 0.22 & 1.44 \\
\hline $2-1,111.5$ & 2.805 & 10.6 & 0.26 & 1.43 \\
\hline $2-1,121.0$ & 2.900 & 14.8 & -0.18 & 1.19 \\
\hline $2-1,131.5$ & 3.005 & 36.1 & -0.78 & 1.04 \\
\hline $2-1,141.0$ & 3.100 & 26.3 & 0.17 & 1.29 \\
\hline $2-2,1.5$ & 3.205 & 23.4 & -0.18 & 1.30 \\
\hline $2-2,11.0$ & 3.300 & 19.4 & -0.57 & 1.40 \\
\hline $2-2,21.5$ & 3.405 & 22.1 & -1.55 & 1.05 \\
\hline $2-2,31.0$ & 3.500 & 28.3 & -1.10 & 1.41 \\
\hline $2-2,41.5$ & 3.605 & 33.0 & -0.87 & 1.27 \\
\hline $2-2,51.0$ & 3.700 & 24.9 & -0.87 & 1.43 \\
\hline $2-2,51.0$ & 3.700 & 24.9 & -1.03 & 1.41 \\
\hline $2-2,61.5$ & 3.805 & 38.2 & -1.27 & 1.01 \\
\hline $2-2,71.0$ & 3.900 & 29.1 & -1.32 & 1.49 \\
\hline $2-2,81.5$ & 4.005 & 29.4 & -0.70 & 1.13 \\
\hline $2-2,89.0$ & 4.080 & 26.3 & -0.03 & 1.19 \\
\hline $2-2,89.0$ & 4.080 & 26.3 & -0.86 & 1.36 \\
\hline $2-2,89.0$ & 4.080 & 26.3 & -0.41 & 1.26 \\
\hline $2-2,101.5$ & 4.205 & 25.2 & -1.21 & 1.43 \\
\hline $2-2,101.5$ & 4.205 & 25.2 & -0.25 & 1.46 \\
\hline $2-2,111.0$ & 4.300 & 31.7 & -1.28 & 1.18 \\
\hline $2-3,2.5$ & 4.715 & 25.6 & 0.13 & 1.26 \\
\hline $2-3,2.5$ & 4.715 & 25.6 & 0.06 & 1.26 \\
\hline $2-3,11.5$ & 4.805 & 24.1 & -0.51 & 0.93 \\
\hline $2-3,21.0$ & 4.900 & 23.8 & -0.57 & 0.99 \\
\hline $2-3,31.5$ & 5.005 & 29.7 & -0.50 & 1.03 \\
\hline $2-3,41.0$ & 5.100 & 28.4 & -0.58 & 1.39 \\
\hline $2-3,51.5$ & 5.205 & 22.2 & -0.30 & 1.62 \\
\hline $2-3,61.0$ & 5.300 & 30.2 & -0.48 & 1.50 \\
\hline $2, \mathrm{CC}, 6.5$ & 5.405 & 25.1 & -0.66 & 1.56 \\
\hline $3-1,11.5$ & 6.215 & 38.1 & -1.09 & 1.72 \\
\hline $3-1,21.0$ & 6.310 & 30.3 & -0.77 & 1.37 \\
\hline
\end{tabular}

Table 1. (Continued).

\begin{tabular}{|c|c|c|c|c|}
\hline \multirow[b]{2}{*}{$\begin{array}{c}\text { Sample } \\
\text { (level in } \mathrm{cm} \text { ) }\end{array}$} & \multirow{2}{*}{$\begin{array}{c}\text { Corrected } \\
\text { Sub-bottom } \\
\text { Depth (m) }\end{array}$} & \multirow{2}{*}{$\begin{array}{c}\text { Coarse } \\
\text { Fraction } \\
(\%)\end{array}$} & \multicolumn{2}{|c|}{ G. sacculifer } \\
\hline & & & $\begin{array}{l}{ }^{18} \mathrm{O} \\
(\%)\end{array}$ & $\begin{array}{l}{ }^{13} \mathrm{C} \\
(\%)\end{array}$ \\
\hline \multicolumn{5}{|c|}{ Hole 502B (cont.) } \\
\hline $3-1,31.5$ & 6.415 & 35.9 & -0.97 & 1.47 \\
\hline $3-1,41.0$ & 6.510 & 26.1 & -1.47 & 1.17 \\
\hline $3-1,51.5$ & 6.615 & 16.5 & -1.18 & 0.74 \\
\hline $3-1,61.0$ & 6.710 & 19.3 & -0.13 & 1.11 \\
\hline $3-1,71.5$ & 6.815 & 17.5 & -0.22 & 1.39 \\
\hline $3-1,81.0$ & 6.910 & 24.0 & -0.16 & 1.28 \\
\hline $3-1,91.5$ & 7.015 & 18.7 & -0.17 & 1.65 \\
\hline $3-1,101.0$ & 7.110 & 23.8 & -0.70 & 1.56 \\
\hline $3-1,112.0$ & 7.220 & 20.1 & -0.32 & 1.36 \\
\hline $3-1,121.0$ & 7.310 & 18.8 & -0.40 & 1.48 \\
\hline $3-1,121.0$ & 7.310 & 18.8 & -0.80 & 1.52 \\
\hline $3-1,131.5$ & 7.415 & 16.2 & -1.06 & 1.60 \\
\hline $3-1,142.0$ & 7.520 & 22.2 & -1.47 & 0.62 \\
\hline $3-2,1.5$ & 7.615 & 22.9 & -0.86 & 1.58 \\
\hline $3-2,11.0$ & 7.710 & 39.3 & -0.95 & 1.39 \\
\hline $3-2,21.5$ & 7.815 & 6.5 & -0.64 & 1.14 \\
\hline $3-2,31.0$ & 7.910 & 17.9 & -0.13 & 1.30 \\
\hline $3-2,41.5$ & 8.015 & 15.5 & 0.41 & 1.36 \\
\hline $3-2,41.5$ & 8.015 & 15.5 & 0.29 & 1.33 \\
\hline $3-2,41.5$ & 8.015 & 15.5 & 0.52 & 1.44 \\
\hline $3-2,51.0$ & 8.110 & 24.1 & -0.03 & 1.63 \\
\hline $3-2,61.5$ & 8.215 & 25.7 & -0.21 & 1.67 \\
\hline $3-2,61.5$ & 8.215 & 25.7 & -0.42 & 1.45 \\
\hline $3-2,71.0$ & 8.310 & 25.4 & -0.27 & 1.80 \\
\hline $3-2,81.5$ & 8.415 & 15.5 & -0.65 & 1.26 \\
\hline $3-2,91.0$ & 8.510 & 15.6 & 0.0 & 1.63 \\
\hline $3-2,101.0$ & 8.610 & 16.4 & -0.63 & 2.25 \\
\hline $3-2,111.5$ & 8.715 & 21.2 & -0.66 & 2.19 \\
\hline $3-2,121.5$ & 8.815 & 29.6 & -1.04 & 1.80 \\
\hline $3-2,131.0$ & 8.910 & 25.6 & -0.68 & 1.89 \\
\hline $3-2,131.0$ & 8.910 & 25.6 & -0.84 & 1.85 \\
\hline $3-2,141.5$ & 9.015 & 26.2 & -0.72 & 1.97 \\
\hline $3-3,1.5$ & 9.115 & 27.7 & -0.50 & 1.91 \\
\hline $3-3,11.5$ & 9.215 & 29.7 & -0.84 & 1.78 \\
\hline $3-3,21.0$ & 9.310 & 28.0 & -0.25 & 1.93 \\
\hline $3-3,31.5$ & 9.415 & 28.7 & -0.49 & 1.88 \\
\hline $3-3,41.0$ & 9.510 & 34.6 & -0.61 & 1.94 \\
\hline $3-3,51.5$ & 9.615 & 26.9 & -0.45 & 2.09 \\
\hline $3-3,61.0$ & 9.710 & 29.3 & -0.44 & 1.72 \\
\hline $3-3,71.5$ & 9.815 & 32.0 & -0.46 & 1.75 \\
\hline $3-3,71.5$ & 9.815 & 32.0 & -0.46 & 1.80 \\
\hline $3-3,83.0$ & 9.930 & 40.9 & 0.06 & 1.57 \\
\hline $3-3,91.5$ & 10.015 & 23.6 & -0.23 & 1.69 \\
\hline $3-3,101.0$ & 10.110 & 25.9 & -0.53 & 1.34 \\
\hline $3-3,112.0$ & 10.220 & 21.8 & -0.26 & 1.54 \\
\hline $3-3,121.0$ & 10.310 & 24.1 & -0.05 & 1.54 \\
\hline $3, \mathrm{CC}, 3.5$ & 10.405 & 21.6 & 0.57 & 1.75 \\
\hline $4-1,11.5$ & 10.655 & 24.6 & -0.27 & 2.05 \\
\hline $4-1,21.5$ & 10.755 & 20.5 & -0.35 & 1.81 \\
\hline $4-1,31.5$ & 10.855 & 24.2 & -0.93 & 1.50 \\
\hline $4-1,41.0$ & 10.950 & 25.2 & -0.94 & 1.43 \\
\hline $4-1,51.5$ & 11.055 & 25.3 & -0.66 & 1.67 \\
\hline $4-1,51.5$ & 11.055 & 25.3 & -1.07 & 1.66 \\
\hline $4-1,61.0$ & 11.160 & 16.8 & -0.29 & 1.50 \\
\hline $4-1,71.5$ & 11.265 & 24.6 & -1.12 & 1.45 \\
\hline $4-1,79.0$ & 11.330 & 21.3 & -1.09 & 1.37 \\
\hline $4-1,91.5$ & 11.455 & 39.3 & -0.91 & 1.45 \\
\hline $4-1,101.0$ & 11.550 & 31.0 & -0.38 & 1.06 \\
\hline $4-1.111 .5$ & 11.655 & 24.0 & 0.19 & 1.36 \\
\hline $4-1,121.0$ & 11.760 & 27.7 & 0.32 & 1.08 \\
\hline $4-1,131.5$ & 11.865 & 32.9 & 0.27 & 1.10 \\
\hline $4-1,139.0$ & 11.930 & 17.0 & 0.27 & 1.05 \\
\hline $4-2,1.5$ & 12.055 & 15.4 & 0.20 & 1.02 \\
\hline $4-2,11.0$ & 12.150 & 19.3 & -0.02 & 0.92 \\
\hline $4-2,21.5$ & 12.255 & 12.0 & -0.29 & 1.24 \\
\hline
\end{tabular}


Table 1. (Continued).

\begin{tabular}{ccccc}
\hline & Corrected & Coarse & \multicolumn{2}{c}{ G. sacculifer } \\
Sample & Sub-bottom & Fraction & ${ }^{18} \mathrm{O}$ & ${ }^{13} \mathrm{C}$ \\
(level in cm) & Depth (m) & $(\%)$ & $(\%)$ & $(\% 0)$ \\
\hline
\end{tabular}

\section{Hole 502B (cont.)}

\begin{tabular}{|c|c|c|c|c|}
\hline $4-2,31.0$ & 12.350 & 15.3 & -0.15 & 1.11 \\
\hline $4-2,41.5$ & 12.455 & 19.9 & -0.39 & 1.42 \\
\hline $4-2,51.0$ & 12.550 & 22.7 & -0.64 & 1.27 \\
\hline $4-2,61.5$ & 12.655 & 21.5 & -0.76 & 1.81 \\
\hline $4-2,71.0$ & 12.750 & 24.5 & -0.36 & 1.68 \\
\hline $4-2,81.5$ & 12.855 & 22.5 & -0.41 & 1.53 \\
\hline $4-2,81.5$ & 12.855 & 22.5 & -0.45 & 1.48 \\
\hline $4-2,91.0$ & 12.950 & 35.9 & -0.32 & 1.59 \\
\hline $4-2,101.5$ & 13.055 & 37.1 & -0.45 & 1.60 \\
\hline $4-2,109.0$ & 13.130 & 37.0 & -0.74 & 1.44 \\
\hline $4-2,121.5$ & 13.255 & 28.1 & -0.43 & 1.17 \\
\hline $4-2,131.0$ & 13.350 & 12.5 & -0.46 & 1.22 \\
\hline $4-2,141.5$ & 13.455 & 38.4 & -0.05 & 1.37 \\
\hline $4-3,1.5$ & 13.555 & 40.0 & 0.19 & 1.58 \\
\hline $4-3,11.5$ & 13.655 & 23.8 & -0.17 & 1.64 \\
\hline $4-3,21.0$ & 13.750 & 23.7 & -0.39 & 1.40 \\
\hline $4-3,31.5$ & 13.855 & 27.3 & -0.23 & 1.52 \\
\hline $4-3,41.0$ & 13.950 & 36.6 & 0.25 & 1.28 \\
\hline $4-3,51.5$ & 14.055 & 28.6 & 0.15 & 1.18 \\
\hline $4-3,61.0$ & 14.150 & 24.2 & 0.03 & 1.36 \\
\hline $4-3,71.5$ & 14.255 & 21.7 & 0.19 & 1.39 \\
\hline $4-3,79.0$ & 14.330 & 26.4 & -0.55 & 1.21 \\
\hline $4-3,91.5$ & 14.455 & 28.5 & -0.98 & 1.53 \\
\hline $4-3,91.5$ & 14.455 & 28.5 & -0.51 & 1.34 \\
\hline $4-3,101.0$ & 14.550 & 29.3 & -0.91 & 1.48 \\
\hline $4-3,101.0$ & 14.550 & 29.3 & -0.65 & 1.54 \\
\hline $4-3,111.5$ & 14.655 & 19.8 & -0.75 & 1.40 \\
\hline $4-3,121.0$ & 14.750 & 22.3 & -0.84 & 1.23 \\
\hline $4, C C, 4.5$ & 14.815 & 29.5 & -0.49 & 1.25 \\
\hline $5-1,21.5$ & 15.015 & 24.2 & -0.55 & 1.53 \\
\hline $5-1,31.5$ & 15.115 & 28.1 & -0.35 & 1.32 \\
\hline $5-1,41.5$ & 15.215 & 24.1 & -0.47 & 1.74 \\
\hline $5-1,51.5$ & 15.315 & 26.7 & -0.77 & 1.46 \\
\hline $5-1,59.0$ & 15.390 & 18.4 & -0.64 & 1.38 \\
\hline $5-1,71.5$ & 15.515 & 33.4 & -0.43 & 1.46 \\
\hline $5-1,83.0$ & 15.630 & 26.7 & -0.75 & 1.58 \\
\hline $5-1,83.0$ & 15.630 & 26.7 & -0.74 & 1.46 \\
\hline $5-1,91.5$ & 15.715 & 29.3 & -0.77 & 1.09 \\
\hline $5-1,101.0$ & 15.810 & 22.1 & -0.54 & 1.25 \\
\hline $5-1,111.5$ & 15.915 & 36.6 & -0.99 & 1.27 \\
\hline $5-1,121.0$ & 16.010 & 34.1 & -0.76 & 0.93 \\
\hline $5-1,131.5$ & 16.115 & 25.7 & -0.76 & 1.25 \\
\hline $5-1,135.0$ & 16.150 & 22.4 & -0.68 & 1.30 \\
\hline $5-1,141.5$ & 16.215 & 24.8 & -0.90 & 0.92 \\
\hline $5-2,1.5$ & 16.315 & 8.3 & -0.91 & 0.97 \\
\hline $5-2,14.0$ & 16.440 & 11.5 & -0.44 & 1.16 \\
\hline $5-2,24.5$ & 16.545 & 9.0 & -0.12 & 1.10 \\
\hline $5-2,24.5$ & 16.545 & 9.0 & 0.0 & 1.00 \\
\hline $5-2,34.0$ & 16.640 & 9.0 & 0.20 & 1.13 \\
\hline $5-2,34.0$ & 16.640 & 9.0 & 0.01 & 1.05 \\
\hline $5-2,44.5$ & 16.745 & 8.4 & -0.06 & 1.00 \\
\hline $5-2,53.0$ & 16.830 & 12.7 & -0.23 & 1.16 \\
\hline $5-2,63.5$ & 16.935 & 20.2 & -0.20 & 1.33 \\
\hline $5-2,71.0$ & 17.010 & 29.1 & 0.24 & 1.37 \\
\hline $5-2,95.5$ & 17.085 & 23.4 & -0.37 & 1.12 \\
\hline $5-2,107.0$ & 17.200 & 28.5 & -0.24 & 1.41 \\
\hline $5-2.121 .5$ & 17.245 & 25.4 & -0.29 & 1.27 \\
\hline $5-2,131.0$ & 17.340 & 26.2 & -0.25 & 1.07 \\
\hline $5-2,131.0$ & 17.340 & 26.2 & -0.47 & 1.39 \\
\hline $5-2,141.5$ & 17.445 & 22.3 & -0.72 & 0.97 \\
\hline $5-3,2.5$ & 17.555 & 20.2 & -0.51 & 1.04 \\
\hline $5-3,11.5$ & 17.645 & 17.6 & -0.61 & 0.95 \\
\hline $5-3,11.5$ & 17.645 & 17.6 & -0.37 & 1.01 \\
\hline $5-3,21.0$ & 17.740 & 20.9 & -0.13 & 0.99 \\
\hline $5-3,31.5$ & 17.845 & 26.5 & -0.63 & 1.30 \\
\hline
\end{tabular}

Table 1. (Continued).

\begin{tabular}{|c|c|c|c|c|}
\hline \multirow[b]{2}{*}{$\begin{array}{c}\text { Sample } \\
\text { (level in } \mathrm{cm} \text { ) }\end{array}$} & \multirow{2}{*}{$\begin{array}{l}\text { Corrected } \\
\text { Sub-bottom } \\
\text { Depth (m) }\end{array}$} & \multirow{2}{*}{$\begin{array}{l}\text { Coarse } \\
\text { Fraction } \\
\quad(\%)\end{array}$} & \multicolumn{2}{|c|}{ G. sacculifer } \\
\hline & & & $\begin{array}{l}{ }^{18} \mathrm{O} \\
(\%)\end{array}$ & $\begin{array}{l}{ }^{13} \mathrm{C} \\
(\%)\end{array}$ \\
\hline \multicolumn{5}{|c|}{ Hole 502B (cont.) } \\
\hline $5-3,41.0$ & 17.940 & 20.1 & -0.91 & 1.42 \\
\hline $5-3,51.5$ & 18.045 & 12.8 & -0.85 & 1.42 \\
\hline $5-3,61.0$ & 18.140 & 13.8 & -1.12 & 1.08 \\
\hline $5-3,71.5$ & 18.245 & 21.7 & -1.00 & 1.57 \\
\hline $5-3,71.5$ & 18.245 & 21.7 & -1.07 & 1.46 \\
\hline $5-3,79.0$ & 18.320 & 27.4 & -0.58 & 1.31 \\
\hline $5-3,79.0$ & 18.320 & 27.4 & -0.57 & 1.27 \\
\hline $5-3,79.0$ & 18.320 & 27.4 & -0.28 & 1.10 \\
\hline $5-3,79.0$ & 18.320 & 27.4 & -0.85 & 1.68 \\
\hline $5-3,79.0$ & 18.320 & 27.4 & -0.66 & 1.11 \\
\hline $5-3,91.5$ & 18.445 & 17.5 & -0.75 & 1.63 \\
\hline $5-3,91.5$ & 18.445 & 17.5 & -0.95 & 1.71 \\
\hline $5-3,99.0$ & 18.520 & 12.5 & -0.95 & 1.53 \\
\hline $5-3,111.5$ & 18.645 & 15.2 & -0.82 & 1.56 \\
\hline $5-3,121.0$ & 18.740 & 28.7 & -0.98 & 1.14 \\
\hline $5, \mathrm{CC}, 3.5$ & 18.825 & 0.0 & -0.44 & 1.59 \\
\hline $5, \mathrm{CC}, 3.5$ & 18.825 & 0.0 & -0.83 & 1.55 \\
\hline $6-1,1.5$ & 19.365 & 10.6 & -0.10 & 1.06 \\
\hline $6-1,11.5$ & 19.465 & 19.1 & -0.30 & 1.62 \\
\hline $6-1,21.0$ & 19.560 & 20.9 & -0.44 & 1.44 \\
\hline $6-1,31.5$ & 19.665 & 19.1 & -0.71 & 1.55 \\
\hline $6-1,41.0$ & 19.760 & 15.8 & -0.81 & 1.51 \\
\hline $6-1,51.5$ & 19.865 & 18.0 & -0.96 & 1.45 \\
\hline $6-1,61.0$ & 19.960 & 20.0 & -0.71 & 1.73 \\
\hline $6-1,71.5$ & 20.065 & 29.1 & -0.62 & 1.32 \\
\hline $6-1,81.0$ & 20.160 & 33.9 & -0.63 & 1.74 \\
\hline $6-1,91.5$ & 20.265 & 26.4 & -0.40 & 1.23 \\
\hline $6-1,101.0$ & 20.360 & 27.9 & -0.36 & 1.31 \\
\hline $6-1,111.5$ & 20.465 & 25.8 & -0.90 & 0.98 \\
\hline $6-1,111.5$ & 20.465 & 25.8 & -0.86 & 1.01 \\
\hline $6-1,121.0$ & 20.560 & 20.5 & -0.39 & 1.52 \\
\hline $6-1,131.5$ & 20.665 & 19.4 & -0.83 & 1.62 \\
\hline $6-1,139.0$ & 20.740 & 17.1 & -0.67 & 1.94 \\
\hline $6-1,150.5$ & 20.855 & 23.5 & -1.15 & 1.57 \\
\hline $6-2,11.0$ & 20.970 & 36.3 & -0.97 & 1.75 \\
\hline $6-2,24.5$ & 21.105 & 49.2 & -1.09 & 1.80 \\
\hline $6-2,31.0$ & 21.150 & 42.4 & -0.70 & 1.50 \\
\hline $6-2,41.5$ & 21.275 & 34.9 & -0.96 & 1.68 \\
\hline $6-2,51.0$ & 21.350 & 32.0 & -0.57 & 1.57 \\
\hline $6-2,51.0$ & 21.350 & 32.0 & -0.70 & 1.49 \\
\hline $6-2,61.5$ & 21.475 & 26.9 & -0.67 & 1.40 \\
\hline $6-2,71.0$ & 21.550 & 24.5 & -0.77 & 1.61 \\
\hline $6-2,81.5$ & 21.675 & 28.9 & -0.84 & 1.47 \\
\hline $6-2,91.0$ & 21.750 & 31.9 & -0.56 & 1.40 \\
\hline $6-2,101.5$ & 21.875 & 31.9 & -0.03 & 1.20 \\
\hline $6-2,109.0$ & 21.930 & 36.0 & -0.37 & 1.25 \\
\hline $6-2,109.0$ & 21.930 & 36.0 & -0.54 & 1.55 \\
\hline $6-3,1.5$ & 22.355 & 21.7 & -0.52 & 1.64 \\
\hline $6-3,11.5$ & 22.455 & 17.5 & -0.68 & 1.51 \\
\hline $6-3,21.0$ & 22.550 & 19.6 & -1.05 & 1.87 \\
\hline $6-3,31.5$ & 22.655 & 21.5 & -1.03 & 1.62 \\
\hline $6-3,41.0$ & 22.750 & 33.4 & -0.84 & 1.65 \\
\hline $6-3,51.5$ & 22.855 & 31.9 & -1.00 & 1.31 \\
\hline $6-3,51.5$ & 22.855 & 31.9 & -1.04 & 1.53 \\
\hline $6-3,61.0$ & 22.950 & 32.7 & -1.04 & 1.68 \\
\hline $6-3,71.5$ & 23.055 & 42.9 & -0.83 & 1.63 \\
\hline $6-3,78.0$ & 23.120 & 27.6 & -0.74 & 1.28 \\
\hline $6-3,91.5$ & 23.255 & 29.8 & -0.54 & 1.58 \\
\hline $6-3,101.0$ & 23.350 & 26.1 & -0.67 & 1.56 \\
\hline $6-3,111.5$ & 23.455 & 23.2 & -0.50 & 2.02 \\
\hline $6-3,119.0$ & 23.530 & 25.1 & -0.57 & 1.32 \\
\hline $6-3,131.5$ & 23.645 & 20.3 & -0.50 & 1.64 \\
\hline $7-1,23.0$ & 23.980 & 34.6 & -0.47 & 1.31 \\
\hline $7-1,23.0$ & 23.980 & 34.6 & -0.33 & 1.03 \\
\hline $7-1,23.0$ & 23.980 & 34.6 & -0.23 & 1.55 \\
\hline
\end{tabular}


Table 1. (Continued).

\begin{tabular}{|c|c|c|c|c|}
\hline \multirow[b]{2}{*}{$\begin{array}{c}\text { Sample } \\
\text { (level in } \mathrm{cm} \text { ) }\end{array}$} & \multirow{2}{*}{$\begin{array}{l}\text { Corrected } \\
\text { Sub-bottom } \\
\text { Depth (m) }\end{array}$} & \multirow{2}{*}{$\begin{array}{c}\text { Coarse } \\
\text { Fraction } \\
(\%)\end{array}$} & \multicolumn{2}{|c|}{ G. sacculifer } \\
\hline & & & $\begin{array}{l}{ }^{18} \mathrm{O} \\
(\%)\end{array}$ & $\begin{array}{l}{ }^{13} \mathrm{C} \\
(\%)\end{array}$ \\
\hline \multicolumn{5}{|c|}{ Hole 502B (cont.) } \\
\hline $7-1,23.0$ & 23.980 & 34.6 & -0.21 & 1.51 \\
\hline $7-2,11.0$ & 24.130 & 16.5 & -0.82 & 1.56 \\
\hline $7-2,31.0$ & 24.330 & 19.2 & -0.94 & 1.34 \\
\hline $7-2,51.0$ & 24.530 & 34.1 & -0.80 & 0.90 \\
\hline $7-2,71.0$ & 24.730 & 32.2 & -0.26 & 1.46 \\
\hline $7-2,91.0$ & 24.930 & 19.7 & -0.47 & 1.80 \\
\hline $7-2,109.0$ & 25.110 & 27.8 & -0.71 & 1.70 \\
\hline $7-2,131.0$ & 25.330 & 36.9 & -0.49 & 1.47 \\
\hline $7-2,131.0$ & 25.330 & 36.9 & -0.22 & 1.20 \\
\hline $7-3,21.0$ & 25.730 & 25.7 & -0.91 & 1.92 \\
\hline $7-3,41.0$ & 25.930 & 34.4 & -0.88 & 1.59 \\
\hline $7-3,61.0$ & 26.130 & 47.0 & -0.63 & 1.51 \\
\hline $7-3,81.0$ & 26.330 & 28.9 & -0.42 & 1.90 \\
\hline $7-3,101.0$ & 26.530 & 23.9 & -0.75 & 2.12 \\
\hline $7-3,121.0$ & 26.730 & 34.8 & -0.86 & 1.62 \\
\hline $7-3,139.0$ & 26.910 & 34.4 & -0.52 & 1.64 \\
\hline $7-4,21.0$ & 27.240 & 19.9 & -0.47 & 2.04 \\
\hline $7-4,41.0$ & 27.440 & 21.5 & -1.00 & 1.75 \\
\hline $7-4,61.0$ & 27.640 & 29.2 & -1.09 & 1.85 \\
\hline $7-4,79.0$ & 27.820 & 43.7 & -0.62 & 1.53 \\
\hline $7-4,79.0$ & 27.820 & 43.7 & -0.22 & 1.38 \\
\hline $7-4,101.0$ & 28.040 & 20.5 & -0.67 & 1.68 \\
\hline $7-4,121.0$ & 28.240 & 18.7 & -1.09 & 1.49 \\
\hline $8-1,83.0$ & 28.870 & 25.9 & -1.17 & 1.22 \\
\hline $8-1,83.0$ & 28.870 & 25.9 & -1.19 & 1.64 \\
\hline $8-1,101.0$ & 29.050 & 12.4 & -0.79 & 1.54 \\
\hline $8-1,121.0$ & 29.250 & 20.9 & -1.16 & 1.68 \\
\hline $8-1,141.0$ & 29.450 & 7.2 & -1.34 & 1.70 \\
\hline $8-2,11.0$ & 29.650 & 22.0 & -1.07 & 2.17 \\
\hline $8-2,31.0$ & 29.850 & 25.1 & -1.05 & 1.88 \\
\hline $8-2,91.0$ & 30.070 & 38.0 & -0.42 & 1.57 \\
\hline $8-2,111.0$ & 30.270 & 22.3 & -0.77 & 1.65 \\
\hline $8-2,111.0$ & 30.270 & 22.3 & -0.53 & 1.72 \\
\hline $8-2,131.0$ & 30.470 & 18.6 & -0.87 & 1.92 \\
\hline $8-3,21.0$ & 30.820 & 39.1 & -1.12 & 1.34 \\
\hline $8-3,41.0$ & 31.020 & 37.8 & -0.71 & 1.50 \\
\hline $8-3,61.0$ & 31.220 & 25.2 & -0.32 & 1.52 \\
\hline $8-3,83.0$ & 31.440 & 29.3 & -0.39 & 1.82 \\
\hline $8-3,101.0$ & 31.620 & 25.7 & -0.78 & 1.92 \\
\hline $9-1,61.0$ & 32.970 & 16.3 & -0.75 & 1.87 \\
\hline $9-1,61.0$ & 32.970 & 16.3 & -0.76 & 1.85 \\
\hline $9-1,83.0$ & 33.190 & 31.9 & -1.15 & 1.85 \\
\hline $9-1,101.0$ & 33.370 & 28.6 & -1.13 & 1.70 \\
\hline $9-1,121.0$ & 33.570 & 31.4 & -1.03 & 1.77 \\
\hline $9-1,141.0$ & 33.770 & 19.9 & -1.22 & 1.87 \\
\hline $9-3,11.0$ & 33.970 & 29.9 & -1.36 & 1.75 \\
\hline $9-2,31.0$ & 34.170 & 34.1 & -0.86 & 1.03 \\
\hline $9-2,51.0$ & 34.370 & 35.8 & -0.51 & 1.29 \\
\hline $9-2,71.0$ & 34.570 & 31.8 & -0.13 & 1.46 \\
\hline $9-2,91.0$ & 34.770 & 31.5 & -0.96 & 1.26 \\
\hline $9-2,111.0$ & 34.970 & 48.4 & -1.27 & 1.43 \\
\hline $9-2,131.0$ & 35.100 & 41.1 & -0.93 & 1.03 \\
\hline $9-2,131.0$ & 35.100 & 41.1 & -0.99 & 1.07 \\
\hline $9-3,16.0$ & 35.450 & 45.8 & -0.82 & 0.87 \\
\hline $9-3,41.0$ & 35.640 & 25.4 & -0.91 & 1.20 \\
\hline $9-3,61.0$ & 35.840 & 33.5 & -1.03 & 1.15 \\
\hline \multicolumn{5}{|l|}{ Hole 502} \\
\hline $3-1,10.0$ & 4.86 & 23.4 & -0.66 & 1.96 \\
\hline $3-1,20.0$ & 4.96 & 31.1 & -0.53 & 1.93 \\
\hline $3-1,30.0$ & 5.06 & 22.5 & -0.46 & 1.35 \\
\hline $3-1,49.0$ & 5.25 & 33.7 & -0.83 & 1.49 \\
\hline $3-1,70.0$ & 5.46 & 30.2 & -0.66 & 1.63 \\
\hline $3-1,90.0$ & 5.66 & 29.6 & -0.84 & 1.48 \\
\hline $3-1,109.0$ & 5.85 & 22.0 & -0.69 & 1.11 \\
\hline
\end{tabular}

Table 1. (Continued).

\begin{tabular}{|c|c|c|c|c|}
\hline \multirow[b]{2}{*}{$\begin{array}{c}\text { Sample } \\
\text { (level in } \mathrm{cm} \text { ) }\end{array}$} & \multirow{2}{*}{$\begin{array}{l}\text { Corrected } \\
\text { Sub-bottom } \\
\text { Depth }(\mathrm{m})\end{array}$} & \multirow{2}{*}{$\begin{array}{c}\text { Coarse } \\
\text { Fraction } \\
(\%)\end{array}$} & \multicolumn{2}{|c|}{ G. sacculifer } \\
\hline & & & $\begin{array}{l}{ }^{18} \mathrm{O} \\
(\%)\end{array}$ & $\begin{array}{l}{ }^{13} \mathrm{C} \\
(\%)\end{array}$ \\
\hline \multicolumn{5}{|l|}{ Hole 502 (cont.) } \\
\hline $3-1,130.0$ & 6.06 & 32.5 & -1.01 & 1.18 \\
\hline $3-1,148.0$ & 6.24 & 22.8 & -0.14 & 0.94 \\
\hline $3-2,19.0$ & 6.45 & 30.0 & -0.17 & 1.10 \\
\hline $3-2,40.0$ & 6.66 & 27.7 & 0.01 & 1.22 \\
\hline $9-1,24.0$ & 31.44 & 22.9 & -0.67 & 1.06 \\
\hline $9-1,24.0$ & 31.44 & & -0.76 & 1.28 \\
\hline $9-1,44.0$ & 31.64 & 26.5 & -0.29 & 1.43 \\
\hline $9-1,64.0$ & 31.84 & 26.1 & -0.20 & 1.64 \\
\hline $9-1,84.0$ & 32.04 & 20.3 & -0.44 & 1.94 \\
\hline $9-1,104.0$ & 32.24 & 22.7 & -0.65 & 1.99 \\
\hline $9-1,124.0$ & 32.44 & 23.4 & -0.93 & 1.97 \\
\hline $9-1,144.0$ & 32.64 & 23.9 & -0.32 & 1.72 \\
\hline $9-2,14.0$ & 32.82 & 34.0 & -0.25 & 1.51 \\
\hline $9-2,34.0$ & 33.02 & 33.7 & -0.38 & 1.71 \\
\hline $9-2,54.0$ & 33.22 & 22.0 & -0.62 & 1.86 \\
\hline $9-2,72.0$ & 33.40 & 27.6 & -0.70 & 1.81 \\
\hline $9-2,72.0$ & 33.40 & & -0.73 & 1.46 \\
\hline $9-2,94.0$ & 33.62 & 10.8 & -0.87 & 2.08 \\
\hline $9-2,114.0$ & 33.82 & 50.6 & -1.26 & 1.65 \\
\hline $9-2,134.0$ & 34.02 & 16.9 & -0.63 & 1.89 \\
\hline $9-3,24.0$ & 34.42 & 21.5 & -0.93 & 1.74 \\
\hline $9-3,44.0$ & 34.62 & 35.7 & -0.86 & 1.73 \\
\hline $9-3,64.0$ & 34.77 & 40.4 & -0.51 & 1.21 \\
\hline $9-3,84.0$ & 34.97 & 37.1 & -0.37 & 1.06 \\
\hline $9-3,84.0$ & 34.97 & & -0.32 & 1.45 \\
\hline $10-1,44.0$ & 35.74 & 18.0 & -0.80 & 1.71 \\
\hline $10-1,64.0$ & 35.94 & 42.9 & -0.77 & 1.23 \\
\hline $10-1,84.0$ & 36.14 & 42.5 & -0.49 & 1.13 \\
\hline $10-1,104.0$ & 36.34 & 22.1 & -0.53 & 1.26 \\
\hline $10-1,124.0$ & 36.54 & 23.1 & -0.60 & 1.35 \\
\hline $10-1,144.0$ & 36.74 & 30.0 & -1.27 & 0.63 \\
\hline $10-2,14.0$ & 36.94 & 22.0 & -0.75 & 1.68 \\
\hline $10-2,34.0$ & 37.14 & 33.0 & -0.51 & 1.33 \\
\hline $10-2,34.0$ & 37.14 & & -0.27 & 1.36 \\
\hline $10-2,55.0$ & 37.35 & 28.0 & -1.26 & 1.25 \\
\hline $10-2,72.0$ & 37.52 & 15.5 & -0.87 & 1.63 \\
\hline $10-2,94.0$ & 37.74 & 42.7 & -0.70 & 1.57 \\
\hline $10-2,116.0$ & 37.96 & 38.7 & -0.99 & 1.59 \\
\hline $10-2,134.0$ & 38.14 & 31.7 & -0.75 & 1.44 \\
\hline $10-3,24.0$ & 38.55 & 15.3 & -0.42 & 1.86 \\
\hline $10-3,44.0$ & 38.75 & 15.6 & -0.50 & 1.87 \\
\hline $10-3,64.0$ & 38.95 & 18.9 & -0.40 & 1.99 \\
\hline $10-3,84.0$ & 39.15 & 16.3 & -0.88 & 1.57 \\
\hline $10-3,84.0$ & 39.15 & & -0.90 & 1.77 \\
\hline $10-3,104.0$ & 39.35 & 21.7 & -0.57 & 1.23 \\
\hline $10-3,124.0$ & 39.55 & 18.9 & -0.71 & 1.57 \\
\hline
\end{tabular}

(lighter or more negative $\delta^{18} \mathrm{O}$ ) indicative of low terrestrial ice volume or high temperatures and even-numbered stages representing enriched values indicative of high terrestrial ice volume or low temperatures. I have not used the nomenclature of van Donk (1976) beyond Stage 23, because his sampling interval was inadequate to recover the stratigraphic detail necessary to define stages comparable to previous work.

The late Quaternary (Brunhes chron) of Hole 502B is characterized by isotopic variations of high amplitude $(1.5-2.0 \%)$ and long periodicities $(\sim 100$ k.y.). The Brunhes Chron of Hole 502B contains isotopic Stages 1 to 19 (see Fig. 1 and 2). This sequence of isotopic stages is consistent with the stratigraphy of Shackleton and 
${ }^{18} \mathrm{O}(\%)$ PDB

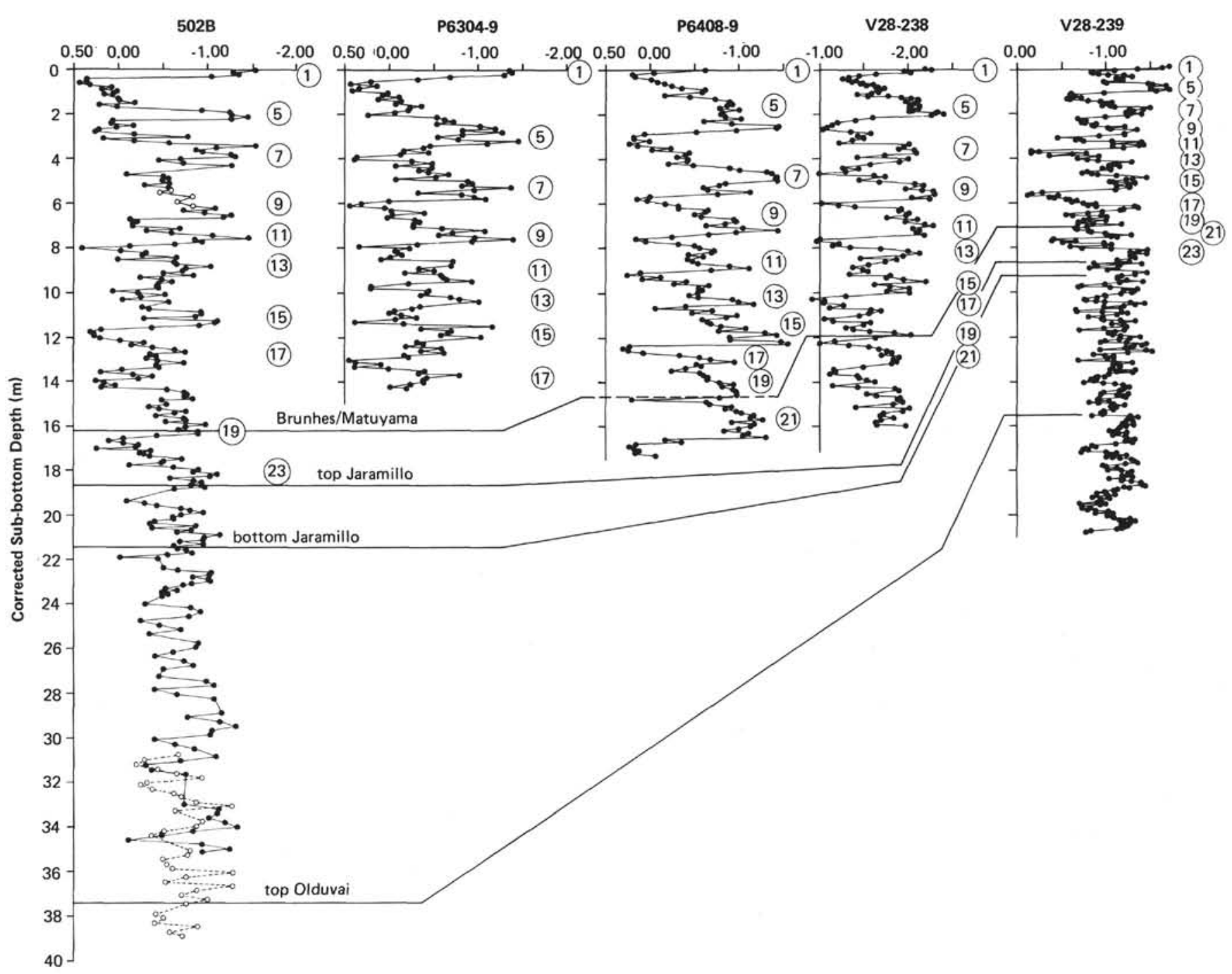

Figure 2. Correlation of oxygen isotopic stratigraphy between the Atlantic (Hole 502B, P6304-9, and P6408-9) and the Pacific (V28-238, V28-239). Interglacial isotopic stage numbers are circles. Where present, magnetostratigraphic boundaries are shown. Cores P6304-9 and P6408-9 are from Emiliani $(1966,1978)$ and Cores Vema 28-238 and Vema 28-239 are from Shackleton and Opdyke $(1973,1976)$.

Opdyke (1973, 1976) and van Donk (1976) and is easily correlated to the stratigraphy in piston cores from the Atlantic and the Pacific (Fig. 2). Although all major stages can be correlated, there are differences in the character of individual stages. For example, the record at Hole 502B does not show the extreme glacial intervals in the early part of the Brunhes of V28-239 (Shackleton and Opdyke, 1976; Pisias and Moore, 1981).

Here, I note that the stages are defined by boundaries which separate "glacial" and "interglacial" intervals. For example, the stages in V28-238 and V28-239 are defined as the depth in core of successive boundaries in the isotopic record (e.g., V28-238, p455, Table 2, Shackleton and Opdyke, 1976). The structure within most stages has not been described as part of the definition of the stage. As a result, boundaries are frequently difficult to define in complex stages (e.g., Stages 17, 18, and 19).
Table 2. The mean and standard deviation of the modes of $\delta^{18} \mathrm{O}$ variation in the early and late Quaternary. 502B data are from this study. Vema 28-238 and Vema 28-239 are from Shackleton and Opdyke (1973, 1976). Data from V16-205 are from van Donk (1976). All measurements are on $G$. sacculifer. All cores show lighter $\delta^{18} \mathrm{O}$ and less variability in the early Quaternary.

\begin{tabular}{lccccc}
\hline & DSDP & & & \\
& Hole & & & \\
& 502B & V28-238 & V28-239 & v16-205 \\
\hline Late Quaternary & $\bar{X}$ & $-0.458 \%$ & $-1.618 \%$ & $-0.978 \%$ & $-0.400 \%$ \\
$(0-730$ k.y.) & $\overline{0}$ & 0.500 & 0.376 & 0.349 & 0.325 \\
Early Quaternary & $\bar{X}$ & $-0.815 \%$ & & $-1.054 \%$ & $-0.643 \%$ \\
$(910-1660$ k.y.) & $\sigma$ & 0.283 & & 0.188 & 0.193 \\
\hline
\end{tabular}

Comparison of the isotopic records in Figure 2 shows that the upper half of Stage 5 is missing in Hole 502B. Apparently, the drill string was lowered about $70 \mathrm{~cm}$ too deep before taking Core 502B-2. We calculate that the 
drilling hiatus extends from near the Stage 4-5 boundary ( $\sim 72$ k.y.) to Substage $5 \mathrm{~d}(\sim 108$ k.y.).

The character of Stages 17, 18, and 19 in 502B also differs from the Pacific cores of Shackleton and Opdyke $(1973,1976)$. Stage 16 is easily identified in all cores as one of the more enriched stages (Fig. 2). Shackleton and Opdyke (1973) defined Stage 17 as the moderately depleted event below Stage 16 . Stage 18 was defined as a complex glacial interval containing two enriched events separated by a short depleted event (not as light as Stage 17). Stage 19 was defined as the relatively short light event which contained the Brunhes/ Matuyama boundary and lies above the enriched interval of Stage 20. In this sequence of stages, the Stage $18-19$ boundary is not especially distinct, because Stage 18 is complex and its lower half not greatly enriched.

In comparison to the Pacific cores, the middle of Stage 18 in 502B is relatively light (i.e., it is as depleted as Stage 17), and the lower part of Stage 18 is not as enriched as the upper part. As a result of these differences, the interglacial interval containing Stage 19 appears to be longer and more square-wave-shaped than it does in the Pacific cores. Other Atlantic cores show similar structure (V22-174, N. J. Shackleton, personal communication) and possibly P6908-9 (Fig. 2), which implies that the difference between Atlantic and Pacific cores may be regionally consistent.

\section{Upper Matuyama Hiatus}

Below the Brunhes Chron, the isotopic stages associated with Jaramillo subchron can be picked with confidence in 502B. The top of the Jaramillo subchron falls in Stage 23, whereas the base falls in Stage 25 (see Shackleton and Opdyke, 1976; van Donk, 1976). This correlation of isotopic and magnetostratigraphy indicates that portions of isotope Stages 20,21, and 22 may be missing in 502B. Comparison of 502B with V28-238 and V28-239 indicates that most of Stage 22 is preserved below the hiatus. This hiatus occurs within Core 5 and is not related to core disturbance or lack or recovery. Other data indicating a hiatus in this interval are the low accumulation rate between the Brunhes/Matuyama and top of the Jaramillo, the low carbonate and coarse fraction between about 16.20 and 17.00 meters sub-bottom, and the presence of micronodules at $\sim 17.0$ meters (Sample $502 \mathrm{~B}-5-2,73 \mathrm{~cm}$ ). The data suggest that erosion or nondeposition caused the hiatus. No increase in benthic/ planktic or fragment/whole-test ratios is observed across this interval, which indicates that increased carbonate dissolution did not cause the hiatus.

\section{Early Quaternary Isotopic Record}

Below the Jaramillo Subchron, the isotopic variation is lower in amplitude and higher in frequency. In this early Quaternary interval, individual stages cannot be as clearly distinguished as in the Brunhes section. Only three records of early Quaternary isotopic variations are available (Site 502, V16-205, and V28-239). Thus the details of the global isotopic variation in this interval remain to be defined. The isotopic record at Hole 502B indicates that distinctive isotopic events may occur near the top of the Olduvai subchron. To resolve the structure of these isotopic events, additional work is needed to decrease the sample interval in the early Quaternary.

\section{DISCUSSION}

\section{Modes of Oxygen Isotopic Variation}

The record of $\delta^{18} \mathrm{O}$ variations in the Quaternary sediments at Site 502 reveals a general pattern of increasing amplitude with time. Examination of the Quaternary record clearly reveals two "modes" of variation. In this context, the term mode is used to indicate a pattern of oceanic variability characteristic of a particular interval. At present a mode is defined by its mean value $(\bar{X})$ and the variance or standard deviation $(\sigma)$ around the mean. With the addition of accurate age models, the distribution of variance as the function of frequency $\left[\sigma_{x}^{2}(f)\right]$ can also be used to define a mode.

In the oxygen isotopic data from Holes 502 and 502B, two modes are apparent: an early Quaternary mode that extends from the top of the Olduvai subchron $(\sim 37.5 \mathrm{~m})$ to the top of the Jaramillo Subchron (18.62 $\mathrm{m})$ and a late Quaternary mode that extends from the base of the Brunhes Chron (16.2 m) to the core top. The early Quaternary mode is characterized by a mean $\delta^{18} \mathrm{O}$ composition of $-0.815 \%$ and a standard deviation $(\sigma)$ of $0.283 \%$ (Tables 2 and 3 ). This mode exhibits relatively light values, low amplitudes, and a noticeable lack of longer period variations. The late Quaternary mode is characterized by a mean $\delta^{18} \mathrm{O}$ composition of $-0.458 \%$ with a standard deviation of $0.50 \%$ (Table 2 ). The late Quaternary mode exhibits both larger amplitude and longer period variations. An $F$ test revealed that the variance of the two $\delta^{18} \mathrm{O}$ modes was significantly different at the 0.95 confidence level. We therefore applied a $t$ test which assumed that the two populations (i.e., modes) had unequal variance (Croxton, 1953; Kendall, 1948) and found that the mean isotopic composition of the modes was also significantly different (0.95).

These data show that the glacial events in the early Quaternary were much less extreme (i.e., there was less terrestrial ice). The data also indicate that interglacial events were less extreme than late Quaternary Stages 1, 5,7 , and 9. Thus early Quaternary interglacial intervals may have contained more terrestrial ice than exists today. Pisias and Moore (1981) reported a similar pattern in Pacific core V28-239. Small changes in sea-surface temperature $\left(\sim 2^{\circ} \mathrm{C}\right)$ could also contribute to this isotopic pattern.

The faster accumulation rate sediments of Hole 502B document for the first time that a significant difference $(\sim 0.4 \%)$ occurs between the mean oxygen isotopic composition of the early Quaternary and the late Quaternary. In the slower accumulation rate piston cores, the difference between early Quaternary and late Quaternary means was small with respect to the precision of the measurements. However, the statistics for V16-205 (van Donk, 1976) and V28-239 (Shackleton and Opdyke, 1976) do show the same pattern as observed in Hole 502B (Table 2). In both of these records, the mean 
$\delta^{18} \mathrm{O}$ of the early Quaternary mode is lighter than the mean of the late Quaternary, although the differences are quite small, especially in the Pacific. The standard deviation of the early Quaternary mode in $502 \mathrm{~B}$ is approximately half that of the late Quaternary mode (Tables 2,3 ). This pattern of decreased variability is also confirmed by the statistics from piston core records (Table 2) and the spectral analysis of V28-239 (Pisias and Moore, 1981).

\section{Transition between Modes}

Although the mean characteristics of a mode can be easily defined, its stratigraphic extent is more difficult to identify. However, transition from the early Quaternary to the late Quaternary mode occurred rapidly. I have selected the transition between interglacial Stage 23 and glacial Stage 22 (near the top of the Jaramillo) as marking the end of the early Quaternary mode. This transition occurs within a relatively short interval, probably not more than 50,000 years. The change in isotopic character at this point in V28-239 has been noted by visual inspection (Shackleton and Opdyke, 1976) and by maximizing the difference in variance between upper and lower sections (Pisias and Moore, 1981). This transition is clearly observed in Hole 502B, V28-239, and V28-238 (see Fig. 2). If this characterization of the transition is correct, it implies that a rapid change in boundary conditions occurred at this time.

Alternatively, Pisias and Moore (1981) have argued that this transition represents the time at which the continental ice sheets became marine-based. They suggest that repeated glacial erosion of the high latitude continents modified the topography to create new seaways and the conditions for marine-based ice caps. Such ice caps could grow larger through the buttressing of marine ice sheets and decay faster. This argument implies that the effects of glacial erosion exceeded a critical level at which the ice caps rapidly changed their response to climatic forcing (i.e., they changed from continental to marine-based ice caps).

\section{Implication for Sea Level Variation}

The similarity between Atlantic and Pacific isotope records indicates that the modes of $\delta^{18} \mathrm{O}$ variation are global rather than regional. Although the statistical characteristics of the modes will vary according to ambient sea-surface temperature and sediment accumulation rate, the basic pattern of lighter oxygen isotopic composition and less variance in the early Quaternary is global and reflects the volume of terrestrial ice. Accordingly, these two modes of $\delta^{18} \mathrm{O}$ variation provide models for the envelope of sea level variability in the early and late Quaternary. Using the calibration of $0.11 \% \delta^{18} \mathrm{O}$ for each 10 meters of sea level change (Fairbanks and Matthews, 1978), the isotopic modes can be translated into sea level variations which are caused by the changing volume of terrestrial ice. The true range of sea level variation is somewhat less than the predicted range, because near-surface temperature changes also contribute to the $\delta^{18} \mathrm{O}$ of Globigerinoides sacculifer.

If modern sea level $(0 \mathrm{~m})$ is initialized to $-1.5 \%{ }_{0} \delta^{18} \mathrm{O}$ (core-top value of $G$. sacculifer), the magnitude of sea level changes relative to modern sea level can be calculated. Here, we note that these relative sea level changes are isotopically derived estimates and do not include the local isostatic effects of hydraulic loading, which could increase the range by up to $30 \%$ (Pitman, 1978). Because two standard deviations around the mean effectively captures the range of the $\delta^{18} \mathrm{O}$ modes, I estimate the envelope of sea level variation to be approximately 182 meters in the late Quaternary and 102 meters in the early Quaternary (Fig. 3). The difference between the mean sea level of the early Quaternary $(-0.82 \%,-62 \mathrm{~m})$ and the late Quaternary $(-0.46 \%,-94 \mathrm{~m})$ suggests that the record of marine transgressions and regressions should be quite different for these two intervals. As a generalization, the early Quaternary should be characterized by eustatic transgressions and regressions in the middle to inner shelf regions, whereas late Quaternary transgressions and regressions should affect the entire shelf, including shelf break in many areas. These two patterns of sea level variation have implications for the

Table 3. Mean and standard deviation of early and late Quaternary isotopic and other oceanic indicators in Hole 502B.

\begin{tabular}{|c|c|c|c|}
\hline & & $\begin{array}{c}\text { Late } \\
\text { Quaternary } \\
\text { (0-730 k.y.) }\end{array}$ & $\begin{array}{c}\text { Early } \\
\text { Quaternary } \\
\text { (910-1660 k.y.) }\end{array}$ \\
\hline$\delta^{18} \mathrm{O}$ & $\underset{\sigma}{\bar{X}}$ & $\begin{array}{c}-0.458 \% \\
0.500\end{array}$ & $\begin{array}{c}-0.815 \% \\
0.283\end{array}$ \\
\hline$\delta^{13} \mathrm{C}$ & $\underset{\sigma}{\bar{X}}$ & $\begin{array}{l}1.401 \% \\
0.275\end{array}$ & $\begin{array}{l}1.560 \% \\
0.313\end{array}$ \\
\hline $\begin{array}{l}\text { Fragment/ } \\
\text { whole tests }\end{array}$ & $\underset{\sigma}{\bar{X}}$ & $\begin{array}{r}15 \\
9\end{array}$ & $\begin{array}{l}25 \\
19\end{array}$ \\
\hline $\begin{array}{l}\% \text { Coarse } \\
\text { fraction }\end{array}$ & $\underset{\sigma}{\bar{X}}$ & $\begin{array}{l}24.4 \% \\
7.0\end{array}$ & $\begin{array}{l}28.8 \% \\
8.4\end{array}$ \\
\hline$\% \mathrm{CaCO}_{3}$ & $\begin{array}{l}\bar{X} \\
n=\end{array}$ & $\begin{array}{l}48.1 \% \\
6.4\end{array}$ & $\begin{array}{l}49.8 \% \\
7.6\end{array}$ \\
\hline
\end{tabular}

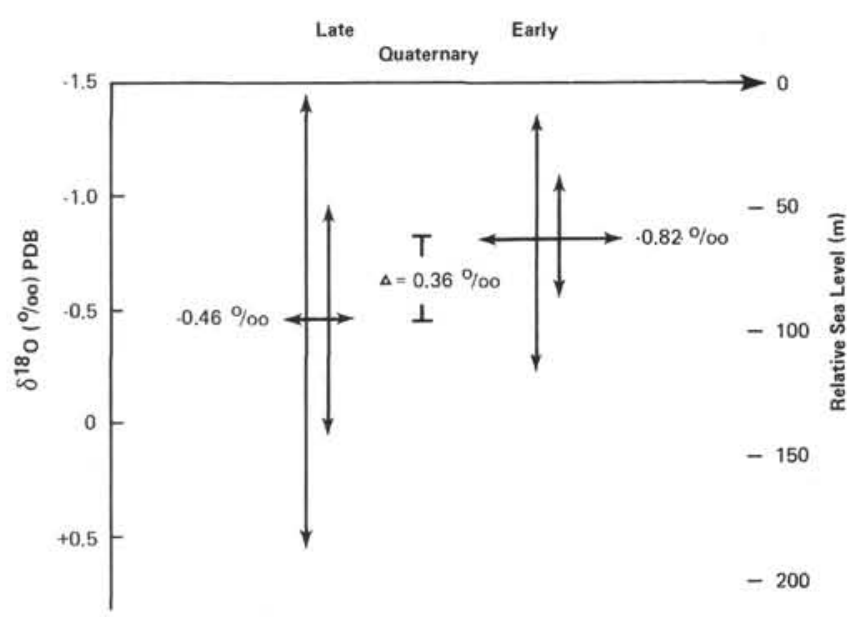

Figure 3. The envelope of sea level variation in the early and late Quaternary as estimated from isotopically derived sea level changes. The calibration of $0.11 \delta^{18} \mathrm{O}$ per each 10 meters of sea level change is from Fairbanks and Matthews (1978). One and two standard deviations around the mean for each mode are shown. The difference in means between early and late Quaternary is equivalent to $33 \mathrm{me}-$ ters sea level change. 


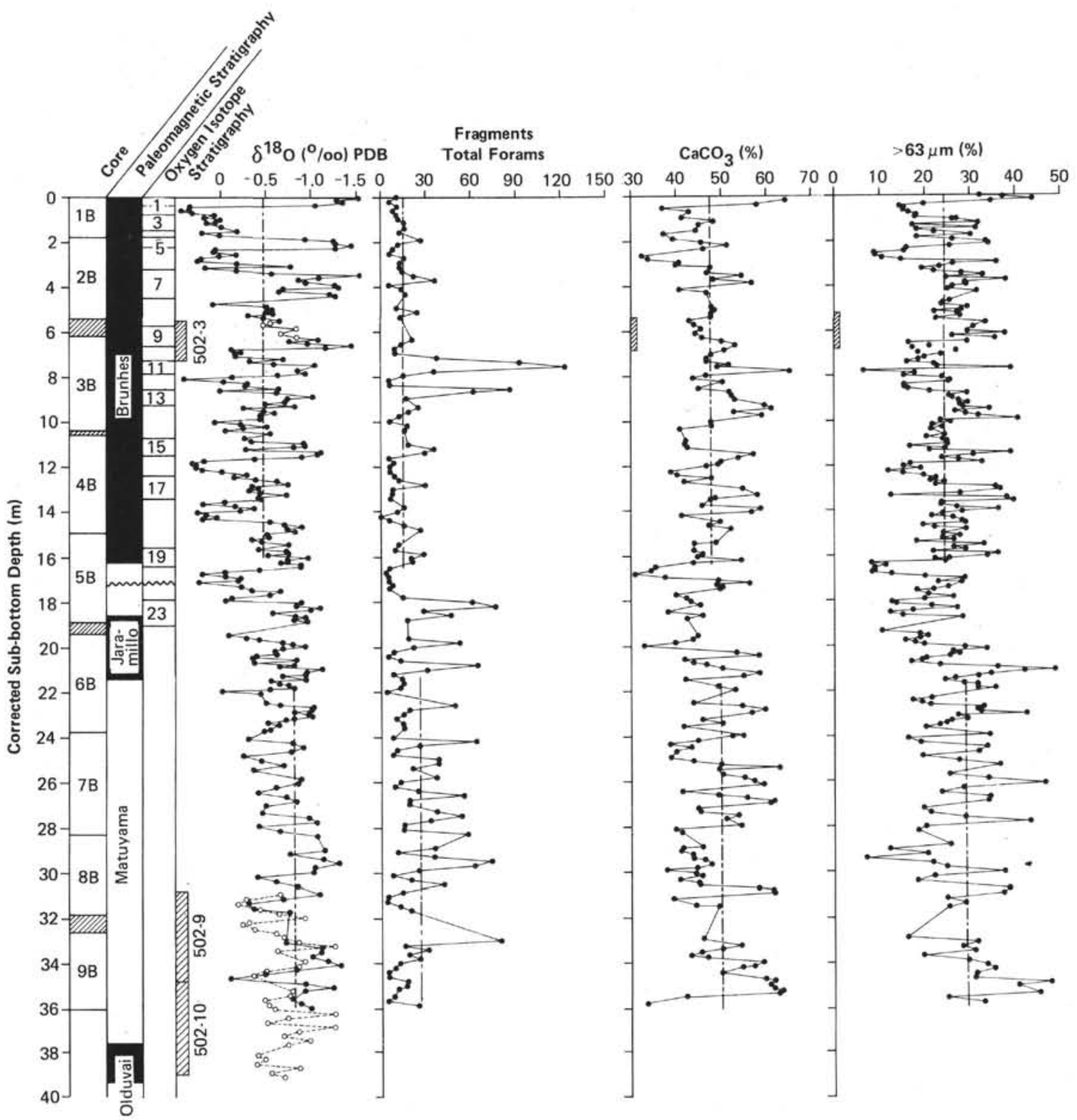

Figure 4. Comparison of oxygen isotope stratigraphy with measures of carbonate dissolution and productivity. The ratio of test fragments to whole foraminiferal tests reflects carbonate dissolution, whereas the percentage of calcium carbonate and coarse fraction $(>63 \mu \mathrm{m})$ reflects productivity, dissolution, and terrigenous dilution. All three measures exhibit different patterns in the early Quaternary and the late Quaternary. However, the fragment ratio displays two clear modes of variation. The mean and standard deviation of the measures are given in Table 3 . All variables change their relationship (phase) with the isotope stratigraphy between early and late Pleistocene.

stratigraphy of continental shelf sediments and the amount of transport off the continental shelf into the deep sea.

\section{Comparing $\delta^{18} \mathbf{O}$ Modes to Other Oceanic Indicators}

Other isotopic and sedimentary parameters also exhibit two modes of variation (Fig. 4). Although the relative differences are less, the mean composition of $\delta^{13} \mathrm{C}$, the foraminiferal content (i.e., coarse fraction $>63$ $\mu \mathrm{m})$, and the ratio of fragments to whole test in the early and late Quaternary are significantly different $(0.99$ confidence level) (Fig. 4, Table 3). Calcium carbonate content is different at the 0.90 level (Table 3 ). Compared to the late Quaternary, the $\delta^{13} \mathrm{C}$ is enriched, and the fragmentation, coarse fraction, and calcium carbonate are higher in the early Quaternary. Although the variances of these parameters are not significantly different from the late Quaternary at the 0.95 level, except for fragmentation, all of the variances are higher in the early Quaternary. These data provide evidence for a mode of variation that is inverse to the $\delta^{18} \mathrm{O}$ pattern. Whereas the mean and variance of $\delta^{13} \mathrm{C}$, coarse fraction, and carbonate tend to decrease from the early to the late Quaternary, the mean and variance of $\delta^{18} \mathrm{O}$ increases. Comparison of these curves with the $\delta^{18} \mathrm{O}$ data suggests that both the amplitude and timing of carbonate dissolution have changed from early to late Quaternary. The fact that oceanic indicators in addition to 
$\delta^{18} \mathrm{O}$ show different modes of variation between the early and the late Quaternary suggests that the boundary conditions between the two periods were substantially different. The temporal relationship between these oceanic indicators and the $\delta^{18} \mathrm{O}$ variations may help elucidate the response of the ice sheets and oceans to climatic change.

\section{CONCLUSIONS}

Examination of the oxygen and carbon isotope stratigraphy and the record of other oceanic indicators for the Quaternary of Holes 502 and 502B reveals the following:

1) The Quaternary section of Holes 502 and 502B is continuous with only a slight hiatus, which occurs in the upper Matuyama. The hiatus is natural and not an artifact of the coring process.

2) The isotopic record of the Brunhes Chron exhibits the standard sequence of 19 oxygen isotope stages.

3) Although the top of the Olduvai Subchron was not recovered in Hole 502B, its depth in that section can be estimated by correlation with the isotopic record of Hole 502, which contains the top of the Olduvai. This approach estimates the top of the Olduvai to be at $\mathbf{3 7 . 5}$ meters in 502B.

4) The character of the $\delta^{18} \mathrm{O}$ record in the early Quaternary and late Quaternary is significantly different. The early Quaternary exhibits a depleted mean composition $\left(-0.82 \%_{0}\right)$ and low variance, whereas the late Quaternary exhibits an enriched mean $(-0.45 \%)$ and high variance. Statistical tests show that both the variance ( $F$ test) and the mean composition (modified $t$ test) of the two modes are significantly different. The data at Site 502 are the first to document the difference in mean oxygen isotopic composition between the early and the late Quaternary.

5) The transition between the two modes is rapid and occurs at about 900,000 years (Pisias and Moore, 1981) at the Stage $22 / 23$ boundary.

6) In terms of ice volume, these modes indicate that the early Pleistocene was characterized by less extensive glacial ice caps than the late Quaternary but somewhat more ice during the interglacials.

7) In terms of sea level variations, mean sea level of the early Quaternary was - 62 meters compared to today and the late Quaternary was -95 meters. These are sea level equivalents not corrected for isostatic rebound. The envelope of sea level variations around these means suggests that the early Quaternary should be characterized by eustatic transgressions and regressions in the middle to inner shelf regions, whereas the late Quaternary should be characterized by transgressions and regressions which affect the entire shelf, including the shelf break.
8) Other oceanic indicators in Hole 502B (carbonate content, coarse-fraction content, and test fragmentation) also indicate two modes of variation, suggesting that the entire oceanic system responded to a change in boundary conditions between the early and the late Quaternary.

\section{ACKNOWLEDGMENTS}

I thank J. J. Morley and T. C. Moore, Jr., for reviewing the manuscript and of fering constructive suggestions and R. K. Matthews for his interest and many discussions of the isotopic data. I gratefully acknowledge A. Hume and C. Savattere for assistance in processing samples, B. Migliore for operation of the mass spectrometer, and C. Sherlock for typing the manuscript. This research was supported by the Climate Dynamics Program, Climate Dynamics Research Section, Division of Atmospheric Sciences; the Seabed Assessment Program, International Decade of Ocean Exploration Section, Division of Ocean Sciences, and the Division of Polar Programs under National Science Foundation grant No. ATM78-25629.

\section{REFERENCES}

Craig, H., 1957. Isotopic standards for carbon and oxygen and correction factors for mass spectrometric analysis of $\mathrm{CO}_{2}$. Geochim. Cosmochim. Acta, 12:133-149.

Croxton, F. E., 1953. Elementary Statistics with Applications in Medicine and the Biological Sciences: New York (Dover Publications, Inc.).

Duplessy, J.-C., Lalou, C., and Vinot, A. C., 1970. Differential isotopic fractionation in benthic foraminifera and paleotemperatures reassessed. Science, 168:250-251.

Emiliani, C., 1955. Pleistocene temperatures. J. Geol., 63:538-573. 1966. Paleotemperature analysis of Caribbean cores P6304. 8 and P6304-9 and a generalized temperature curve for the past 425,000 years. J. Geol., 74:109-124.

1978. The cause of the ice ages. Earth Planet. Sci. Lett., $37: 349-352$.

Fairbanks, R. G., and Matthews, R. K., 1978. The marine oxygen isotope record in Pleistocene coral, Barbados, West Indies. Quat. Res., 10:181-196.

Kendall, M. G., 1948. The Advanced Theory of Statistics: London (Charles Griffin \& Company Limited).

Pisias, N. G., and Moore, T. C., Jr., 1981. The evolution of Pleistocene climate: A time series approach. Earth Planet. Sci. Lett., $52: 450-458$.

Pitman, W. C., III, 1978. Relationship between eustacy and stratigraphic sequences of passive margins. Geol. Soc. Am. Bull., 89: 1389-1403.

Shackleton, N. J., 1967. Oxygen isotope analyses and palaeotemperatures re-assessed. Nature, 215:15-17.

Shackleton, N. J., and Opdyke, N. D., 1976. Oxygen isotope and paleomagnetic stratigraphy of Pacific core V28-239 Late Pliocene to Latest Pleistocene. In Cline, R. M., and Hays, J. D. (Eds.), Investigations of Late Quaternary Paleoceanography and Paleoclimatology: Geol. Soc. Am. Mem., 145:449-464.

1973. Oxygen isotope and palaeomagnetic stratigraphy of equatorial Pacific core V28-238: Oxygen isotope temperatures and ice volumes on a $10^{5}$ and $10^{6}$ year scale. Quat. Res., 3:39-55.

van Donk, J., 1976. $\mathrm{O}^{18}$ record of the Atlantic Ocean for the entire Pleistocene epoch. In Cline, R. M., and Hays, J. D. (Eds.), Investigations of Late Quaternary Paleoceanography and Paleoclimatology: Geol. Soc. Am. Mem., 145:147-163. 